\title{
KINETIC EXCHANGE IN DILUTED MAGNETIC SEMICONDUCTORS OF WURTZITE STRUCTURE*
}

\author{
J. BLINOWSKI \\ Institute of Theoretical Physics, Warsaw University \\ Hoża 69, 00-681 Warszawa, Poland \\ AND P. KaCMAN \\ Institute of Physics, Polish Academy of Sciences \\ Al. Lotników 32/46, 02-668 Warszawa, Poland
}

Kinetic exchange between valence electrons and paramagnetic ions in diluted magnetic semiconductors of wurtzite structure is examined and compared with the results obtained previously for zinc-blende type diluted magnetic semiconductors. Two limiting electron configurations of the impurity ion, $d^{5}$ and $d^{1}$, are discussed. For the former, it is shown that the exchange constant anisotropy, experimentally observed in CdMnSe, results from the anisotropy of the hybridization matrix elements. In the latter case, apart from the similar anisotropy of the ferromagnetic exchange constant, additional, antiferromagnetic corrections should be expected, since for this particular symmetry the hybridization between the ground state of the ion and valence band becomes allowed.

PACS numbers: 75.50.Pp, 75.30.-m

\section{Introduction}

In our previous papers (see [1] and the references therein) we have investigated theoretically the kinetic exchange between $\Gamma_{8}$ valence electrons and tetrahedrally coordinated various paramagnetic ions in zinc-blende type $\mathrm{A}^{I I} \mathrm{~B}^{V I} \mathrm{di}$ luted magnetic semiconductors (DMS). We have shown that the effective kinetic exchange Hamiltonian strongly depends on the electron configuration of the ion ranging from purely ferromagnetic to purely antiferromagnetic Kondo-like Hamiltonian via more complicated forms sensitive to Jahn-Teller distortions. When following the details of our derivation, one observes that these results lean heavily on the particular symmetry of both the crystal potential acting on the paramagnetic

${ }^{*}$ This work was partially supported by the grant of the Committee for Scientific Research 2-09-28-91-01. 
ion and of the valence band states - they are subjected considerably to changes when different symmetries are considered. This is also proved by the experimental data on diluted magnetic semiconductors of wurtzite structure (see e.g., [2]) where the axial anisotropy of the kinetic exchange interactions was detected.

In this paper we examine the kinetic exchange between valence band electrons and the trigonally coordinated paramagnetic ions in $\mathrm{A}^{I I} \mathrm{~B}^{V I}$ DMS of wurtzite lattice. The symmetry induced modifications of the valence band structure, the paramagnetic impurity spectrum and the hybridization matrix elements are taken into account.

\section{Model}

It is well established that for an adequate description of the valence band states in the $\mathrm{A}^{I I} \mathrm{~B}^{V I}$ compounds of wurtzite structure it is enough to consider a group of three bands labeled usually as $A, B, C$. These bands are split by the combined effect of the anisotropic crystal field and the spin-orbit interaction, both treated as perturbations acting on the six-fold degenerated valence band with the basis functions $Z \alpha,(X+\mathrm{i} Y) \alpha / \sqrt{2},(X-\mathrm{i} Y) \alpha / \sqrt{2}$, where $\alpha$ represents the two spin states and the orbital parts are the cubic Bloch functions transforming like the spherical harmonics $Y_{1}^{0}, Y_{1}^{1}$ and $Y_{1}^{-1}$ under the crystal symmetry operations, respectively.

Our objective will be the effective kinetic exchange IIamiltonian representing in the above basis the interactions of a band electron with the paramagnetic impurities. As in the case of zinc-blende DMS we use for ions the spherical Parmenter Hamiltonian, thus neglecting the direct effect of the ligand field on the $d$-orbitals [1]. In the reference system with the origin at the impurity position and $z$-axis along the crystal $c$-axis, the hybridization IIamiltonian has the form

$$
H_{\mathrm{hyb}}=\sum\left[M_{\mu j \sigma} a_{\mu j \sigma}^{+}+\text {h.c. }\right] \text {, }
$$

where $\mu$ numbers the irreducible representations of the point group $C_{3 v}$ under which the one-electron $d$-orbitals transform: $\mu=1$ for $\Gamma_{1}$ and $\mu= \pm 3$ for the two $\Gamma_{3}$ representations; $j$ numbers the basis functions of each representation and $\sigma$ is the spin index. The operator $a_{\mu j \sigma}^{+}$creates an electron with the spin $\sigma$ in the $d$ orbital $\mu j$.

$$
M_{\mu j \sigma}=\left(V_{\mu} / \sqrt{\Omega}\right) a_{\left.\right|_{\mu j} j \boldsymbol{k}_{\sigma}},
$$

where $V_{\mu}$ are nonvanishing matrix elements of the one-electron Hamiltonian $T$ (transforming according to $\Gamma_{1}$ representation of $C_{3 v}$ ) between the Bloch functions at $k=0$ and the atomic $d$-orbitals states $|L=2, M\rangle$.

$$
\begin{aligned}
& V_{1}=\langle 2,0|T| Z\rangle, \\
& V_{3}=\langle 2,1|T|(X+\mathrm{i} Y) / \sqrt{2}\rangle, \\
& V_{-3}=\langle 2,-2|T|(X+\mathrm{i} Y) / \sqrt{2}\rangle .
\end{aligned}
$$

(In the cubic limit with the $z$-axis along [111] crystal direction we would have $\left.V_{1} / \sqrt{3}=-V_{3}=V_{-3} / \sqrt{2}\right) . \Omega$ denotes the volume of the crystal. The operator $a_{\mu^{\mu} j k_{j}}$ annihilates the band electron from the appropriate Luttinger state. 


\section{Kinetic exchange}

The effective kinetic exchange Hamiltonian $H_{p-d}$, which describes the scattering of a band electron on an impurity ion, is obtained within the above model by the canonical transformation method described in detail in Ref. [1]. We restrict our considerations to two extreme electron configurations of the impurity ion: $d^{5}$ - the half filled $d$-shell (e.g., $\mathrm{Mn}^{2+}, \mathrm{Fe}^{3+}$ ) and $d^{1}-$ one electron in the $d$-shell (e.g., $\mathrm{Sc}^{2+}, \mathrm{Ti}^{3+}$ ), known to lead in the cubic case to purely antiferromagnetic and purely ferromagnetic Kondo-like kinetic exchange, respectively. Below we present the mean field effective exchange Hamiltonians for valence electrons interacting with these two kinds of magnetic ions in the case of magnetic field parallel to the $z$-axis.

\section{1. $d^{5}$ ions}

The ground state of the $d^{5}$ ion in the trigonal environment is still an orbital singlet as it is in the cubic case. Thus, the single-ion effective kinetic exchange Hamiltonian may depend only on the spin of the ion. As in the cubic case it has the form of the antiferromagnetic Kondo-like Hamiltonian. In contrast to the cubic case we obtain, however, two different exchange constants: $\beta_{z}$ for $Z \alpha$ states and $\beta_{x}$ for $(X \pm \mathrm{i} Y) \alpha / \sqrt{2}$ states.

$$
\beta_{i}=2 B_{5, i}
$$

where $B_{5, i}$ is given by the formula (19) in [1] with $V^{2}$ replaced by $V_{1}^{2}$ in $\beta_{z}$ and by $\left(V_{3}^{2}+V_{-3}^{2}\right)$ in $\beta_{x}$. One can see that, within our model, for $d^{5}$ ions the anisotropy of the exchange results entirely from the trigonal modifications of the hybridization matrix elements.

The mean-field exchange Hamiltonian, obtained by summing up the thermodynamical a verages of our single-ion exchange Hamiltonians, can be written in the form (9) of Ref. [2] with $\beta_{z}$ and $\beta_{x}$ given by Eq. (4).

\section{2. $d^{1}$ ion}

The wave functions for the ground state doublet $E$ of the $d^{1}$ ion in the trigonal environment can be chosen in the form

$$
|E, \pm\rangle= \pm a|2, \pm 1\rangle \pm \sqrt{1-a^{2}}|2, \mp 2\rangle
$$

where $a$ is a constant, which in the cubic limit (with the $z$-axis along [111] crystal direction) would be equal to $\sqrt{(2 / 3)}$.

In contrast to the cubic case, where the wave functions (5) do not hybridize by symmetry with the $p$-like Bloch functions, here the hybridization is symmetry allowed since $\pm(X \pm \mathrm{i} Y) / \sqrt{2}$ and (5) transform according to the same $E$ representation.

The mean-field effective kinetic exchange Hamiltonian for valence electron interacting with $d^{1}$ ions can be also put in the form (9) of Ref. [2], but this time with the exchange constants

$$
\begin{aligned}
& \beta_{z}=-2 C_{1, z}, \\
& \beta_{x}=-2 C_{1, x}+2\left(C_{1, a}+B_{1, a}\right),
\end{aligned}
$$


where $C_{1, z}$ and $C_{1, x}$ are given by Eq. (21) in [1] with $V^{2}$ replaced by $V_{1}^{2}$ and by $\left(V_{3}^{2}+V_{-3}^{2}\right)$, respectively. To obtain the constants $B_{1, a}$ and $C_{1, a}$ one has to replace $V$ in formulae (19) and (21) in [1] by $\left(a V_{3}+\sqrt{1-a^{2}} V_{-3}\right)$.

One can see that for the $d^{1}$ configuration in trigonal environment the leading ferromagnetic term has a similar anisotropy to that obtained for $d^{5}$ ions. For the bands $(X \pm \mathrm{i} Y) / \sqrt{2}$ we get moreover a small antiferromagnetic correction, which depends on the composition of the ionic orbital ground state and vanishes in the cubic limit (since in this limit $a V_{3}+\sqrt{1-a^{2}} V_{-3}=0$ ).

\section{References}

[1] J. Blinowski, P. Kacman, Phys. Rev. B 46, 12298 (1992).

[2] M. Arciszewska, M. Nawrocki, J. Phys. Chem. Solids 47, 309 (1986). 